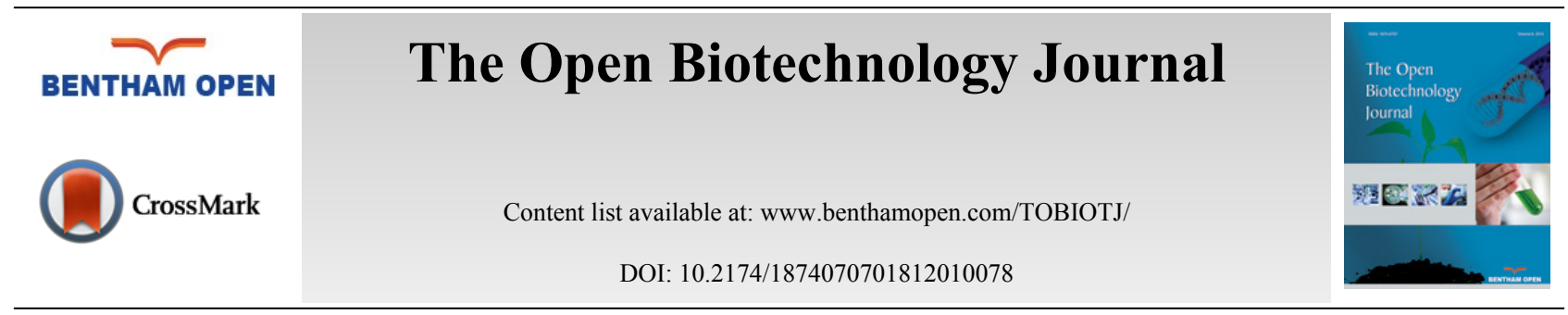

RESEARCH ARTICLE

\title{
Enhancement of Recombinant Antibody Expression Level by Growth Controlled Medium
}

Le Thi Minh Phuc ${ }^{1,2,3}$, Tetsuji Sasaki ${ }^{4}$, Hisayo Shimizu ${ }^{4}$, Nguyen Thi Minh Huyen ${ }^{3}$, Nguyen Thi Thu Thuy $^{3}$, Le Quang Huan ${ }^{3}$ and Akiyoshi Taniguchi ${ }^{1,2, *}$

${ }^{I}$ Cellular Functional Nanomaterials Group, Research Center for Functional Materials, National Institute for Materials Science, 1-1 Namiki, Tsukuba, Ibaraki 305-0044, Japan

${ }^{2}$ Graduate School of Advanced Science and Engineering, Waseda University, 3-4-1 Okubo, Shinjuku-ku, Tokyo 169-8555, Japan

${ }^{3}$ Institute of Biotechnology, Vietnam Academy of Science and Technology, 18 Hoang Quoc Viet, Cau Giay, Hanoi, Vietnam

${ }^{4}$ Kyokuto Pharmaceutical Industrial Co., Ltd. 3333-26 Aza-Asayama, Kamitezuna, Takahagi-shi, Ibaraki 318-0004, Japan

\section{Abstract:}

\section{Background:}

Transient expression system is very widely used for protein expression including recombinant protein expression. Normally, in the commercially available expression system, cells were grown rapidly in protein expression. However, over cell growth was not only unnecessary for high level protein expression, but also induced cell death and led to protein degradation.

\section{Aims and Objectives:}

To overcome these limitations a new adapted culture method needs to be developed.

\section{Methods:}

In this work, we developed growth control medium for transient high protein expression system using NPLAd (Non-Protein and Lipid Medium Adopted) cells. With this system, cell numbers were not increased until 10 days.

Results:

Our results indicated that expression level in NPLAd system was 1.8 times higher than that in Free style system, which was one of the commercially available high expression systems.

\section{Conclusion:}

The antibody performance expressed by NPLAd system was almost the same to that of expressed by Free style system. The results suggested that NPLAd system could be useful for protein expression, such as recombinant antibody.

Keywords: Recombinant antibody, Protein expression, CHO cells, Culture medium, NPLAd system, Antibody performance.

\footnotetext{
*Address correspondence to this author at the Cellular Functional Nanomaterials Group, Research Center for Functional Materials, National Institute for Materials Science, 1-1 Namiki, Tsukuba, Ibaraki 305-0044, Japan; Tel: 81-29-860-4505; E-mail: taniguchi.akiyoshi@nims.go.jp
} 


\section{INTRODUCTION}

Monoclonal antibodies are most selling biopharmaceutics drug in drug market [1 - 4]. Many of the recombinant production systems have been developed, such as gram-negative and positive bacteria, yeasts and filamentous fungi, insect cell lines, mammalian cells to transgenic plants and animals [5]. The advanced mammalian folding, secretion and post-translational apparatus are capable of producing antibodies indistinguishable from those in the human body with least concerns for immunogenic modifications. Moreover, it is also highly efficient for secretion of large and complex IgGs [5]. On the other hand, expression system using mammalian cells has some risk such as viral infections [3 - 7].

Chinese Hamster Ovary (CHO) cells are the prominent choice of mammalian expression system for antibodies [8 10]. Due to safety concerns in clinical use, the expression system of CHO cells should not have biological ingredients, such as serum [11]. The adapted culture method is one of the good strategies to simplify CHO cell culture medium without any proteins and lipids. The strategy of adapted culture method includes both medium and cell modification. However, in biological ingredient-free culture systems, the rate of cell proliferation is decreased. Proliferation in simplified cell culture medium often requires autocrine factors, such as EGF [12]. To increase proliferation, the signaling efficiency of these autocrine factors should be increased. We have developed a Non-Protein and Lipid Medium Adopted (NPLAd) cell line for biopharmaceutical recombinant protein expression [13]. The proliferation rate of CHO cells in simplified culture medium was improved by insulin and GM3 addition. Our results suggest that this cell line could be useful for biopharmaceutical recombinant protein expression. However, NPLAd system has some problems, such as low protein expression level, because NPLAd medium was good for cell growth, but not good for protein expression.

In this paper, we developed growth control medium for transient high protein expression system using NPLAd cells with our new concept. Our concept was that cell growth was not necessary for protein expression in transient expression system because most of the cells were not transfected by expression vector. Keeping this in view, we developed low growth rate medium for antibody expression. The results indicated that expression level in this system was higher than that in conventional system, FreeStyle System, which is one of commercially high expression system. The results suggested that this system could be useful for recombinant antibody expression.

\section{MATERIALS AND METHODS}

\subsection{Cell Culture and Medium}

Free Style Chinese Hamster Ovary (CHO) cell line (FS CHO-S Cells, ThermoFisher Scientific) manipulated to express was used in the study. FreeStyle CHO Expression Medium (ThermoSicher Scientific, USA) was used as the FreeStyle CHO cell growth medium. In addition, an in-house NPLAd cell line, has been previously described [13]. NPL medium, previously described [13] was modified and used as the NPLAd cell line growth medium. The improved NPL medium was named NPLTT medium. Both FreeStyle and NPLAd Systems were inoculated in shaking flask and incubated at $37^{\circ} \mathrm{C}$ under $5 \% \mathrm{CO}_{2}, 100 \%$ humidity and $120 \mathrm{rpm}$ shaking.

The improved NPL medium was named NPLTT medium (NPL Medium for Transient Transfection). The concentration of L-Isoleucine, L-leucine, L-ornithine and L-threonine was increased as a protein expression resource in NPLTT medium. The NPLTT medium increased glucose and mannose concentrations as energy sources for cells. Ascorbic acid and glutathione were added to suppress cell damages by their antioxidant effects.

\subsection{Transfection Efficiency}

Transfection efficiency of transient gene expression was measured by GFP expression assay. Green Fluorescent Protein vector, phMGFP (Promega) was amplified in DH5 $\alpha$ and purified with QIAGEN Plasmid Midi Kit (QIAGEN, USA). Freestyle CHO-S cells (Thermo fisher scientific, USA) were cultured in Freestyle CHO medium (Thermo fisher scientific) supplemented with $2 \mathrm{mM}$ L-glutamine. NPLAd and FreeStyle CHO-S cells were cultured for semi-confluent condition under standard humidified conditions $\left(37^{\circ} \mathrm{C}\right.$ and $\left.5 \% \mathrm{CO}_{2}\right)$ and harvested. Cells were resuspended in fresh medium and seeded in 24-well plates by $20 \times 10^{5}$ cells in $0.5 \mathrm{~mL}$ medium. 3 wells were tested for a cell condition. Culture plates were incubated at $37^{\circ} \mathrm{C}$ and $5 \% \mathrm{CO}_{2}$ in shaking at $120 \mathrm{rpm}$. Transfection reagent Lipofectamine LTX \& PLUS Reagent (Thermo fisher scientific) and Freestyle MAX were used for NPLAd cells and Freestyle CHO-S cells, respectively, under standard protocol. $1 \mu \mathrm{g}$ of phMGFP plasmid was used for a well and Opti-MEM (Thermo fisher scientific) was used to prepare transfection complex. GFP expression was measured after 6 days from transfection day with LUNA-FL Dual Fluorescence Cell Counter (Logos Biosystems, USA). 


\subsection{Plasmid Construction}

Anti-HER2 antibody sequence was inserted into pCDNA3.1 vector between Nhe I and Xho I restriction site. The order of the sequence was as follows: signal peptide, heavy chain (VH), GS repeat region, light chain (VL) and constant regions (CH2-CH3). The signal peptide was a 19-amino-acid sequence (MKHLWFFLLLVAAPRWVLS) the original of which comes from signal peptide of V-region precursor (Genebank: AAA58803). This sequence was designed to be auto-cleavaged after leading the anti-Her2 antibody throughout cell into the culture medium. The auto-cleavage ability of this sequence was tested using SignalP 4.1 Server (http://www.cbs.dtu.dk/services/SignalP/). VH and VL DNA region were constructed following the synthetic sequence of the author's group (Genbank: AM402973.1). Between VH and VL, a 35-GS repeat region was inserted to create a hinge region which makes VH and VL more flexible. The CH2$\mathrm{CH} 3$ region was directly cloned from Vietnamese blood sample.

\subsection{Cell Growth Assay and Recombinant Antibody Expression}

In the NPLAd System, NPLAd cells were seeded at 1 × $10^{6}$ cell / $\mathrm{mL}$ in a Shaking flask. Shaking culture was carried out for 24 hours as preculture. After preculture, $2 \mu \mathrm{L} / \mathrm{mL}$ of FuGENE HD transfection reagent (Promega) and $0.4 \mu \mathrm{g} /$ $\mathrm{mL}$ of anti-HER 2 antibody vector were added. Cultured for 4 hours after transfection, $1 \mathrm{mM}$ of valproic acid was added. Shaking culture was continued for 2 weeks at $37^{\circ} \mathrm{C}$.

In the FreeStyle System, CHO-s cells were seeded at a cell density of $2 \times 10^{5}$ cells $/ \mathrm{mL}$. Transfection was performed by adding $1 \mu \mathrm{g} / \mathrm{mL}$ of Lipofectamine LTX Reagent with PLUS Reagent (Thermo Fisher Scientific) and 0.4 $\mu \mathrm{g} / \mathrm{mL}$ of anti-HER 2 antibody vector. After transfection, shaking culture was carried out for 12 days at $37^{\circ} \mathrm{C}$.

Samples were taken at regular intervals on both systems and the number of cells and the expression levels of antibody were measured. The cell number was measured by a dye exclusion method with Hemocytometer. Antibody concentration was measured by ELISA using human IgG ELISA quantitation set (Bethyl laboratories inc, USA).

\subsection{Purification of Recombinant Antibody}

Antibodies expressed by NPLAd System and FreeStyle System were purified by the same method. Culture supernatant was desalted with a Vivaflow 50 ultrafiltration membrane (Sartorius AG, MWCO 30,000) and concentrated to 250 times.

Desalted and concentrated culture supernatant was purified using a commercially available Antibody Purification Kit Protein A (Bio-Rad) according to kits protocol. After affinity purification, the purity and recovered amount of the antibody were measured. The purity of the antibody after purification was calculated by measuring the amount of antibody by ELISA, measuring the protein concentration with a protein assay kit (Bio-Rad), and dividing the amount of antibody by the amount of protein. The recovery rate of the antibody after purification was calculated by dividing the total amount of antibody in the culture supernatant by the total amount of purified antibody.

Purified antibodies were electrophoresed on SDS-PAGE under non-reducing conditions (without DTT). Approximately, $100 \mathrm{ng}$ of purified antibody per lane is supplied to an electrophoresis gel. After electrophoresis, western blotting was performed. Anti-human IgG-HRP was bound to the blotted nitrocellulose membrane, followed by antibody staining with EzWest Blue (ATTO Corporation). Molecular weight was estimated using Marker as Precision Plus Protein Dual Color Standards (MW range from 10 to $150 \mathrm{kD}$, Bio-Rad).

\subsection{ELISA for Binding Activity of Anti-HER2 Antibody}

Binding activity of anti-HER2 antibody was determined using human IgG ELISA quantitation set (Bethyl laboratories inc) according to the manufacturer's instructions with some modified. The A431 cells were seeded at density $10^{4}$ cells/well in a 96-well plate at $37^{\circ} \mathrm{C}, 24 \mathrm{~h}$. Next, the cells were washed once with wash solution and fixed with $100 \mu 14 \%$ formaldehyde at room temperature (rt), $15 \mathrm{~min}$. Then the cells were washed twice with wash solution and blocked with $200 \mu$ l blocking solution within $30 \mathrm{~min}$ at rt. After removing the blocking solution, cells were washed 5 times and incubated to $100 \mu \mathrm{l}$ of samples. Cells with no incubation to anti-Her2 antibody were also tested as controls. $100 \mu \mathrm{l}$ of diluted HRP labeled antibody was added to each well. After 1 hour incubation, cells were washed 5 times. Following $100 \mu \mathrm{l}$ of TMB substrate solution was added to each well and the plate was developed in the dark for 15 min. The reaction was stopped by adding $100 \mu \mathrm{l} 0.18 \mathrm{M} \mathrm{H}_{2} \mathrm{SO}_{4}$ to each well, absorbance at $450 \mathrm{~nm}$ was measured by a plate reader. 


\section{RESULTS AND DISCUSSION}

\subsection{The Transfection Efficiency of FS CHO-S and NPLAD Cells}

At first, we checked the transfection efficiency of FS CHO-S and NPLAd cells using GFP expression vector. For FS CHO-S cells, Freestyle MAX were used under standard protocol. The transfection efficiency of FS CHO-S was approximately 95\% (Fig. 1A). We also used Freestyle MAX for NPLAd cells, however, transfection efficiency was quite low (data not shown). For NPLAd cells, we checked several kinds of transfection reagents, Lipofectamine LTX \& PLUS reagent showed the highest transfection efficiency among these transfection reagents. The transfection efficiency of NPLAd cells using Lipofectamine LTX \& PLUS was approximately $21 \%$ (Fig. 1B). The results indicated that the transfection efficiency of NPLAd cells was approximately 4.5-fold lower than that of FS CHO-S. Low transfection efficiency could be due to cell membrane lipid component of NPLAd cells [14].
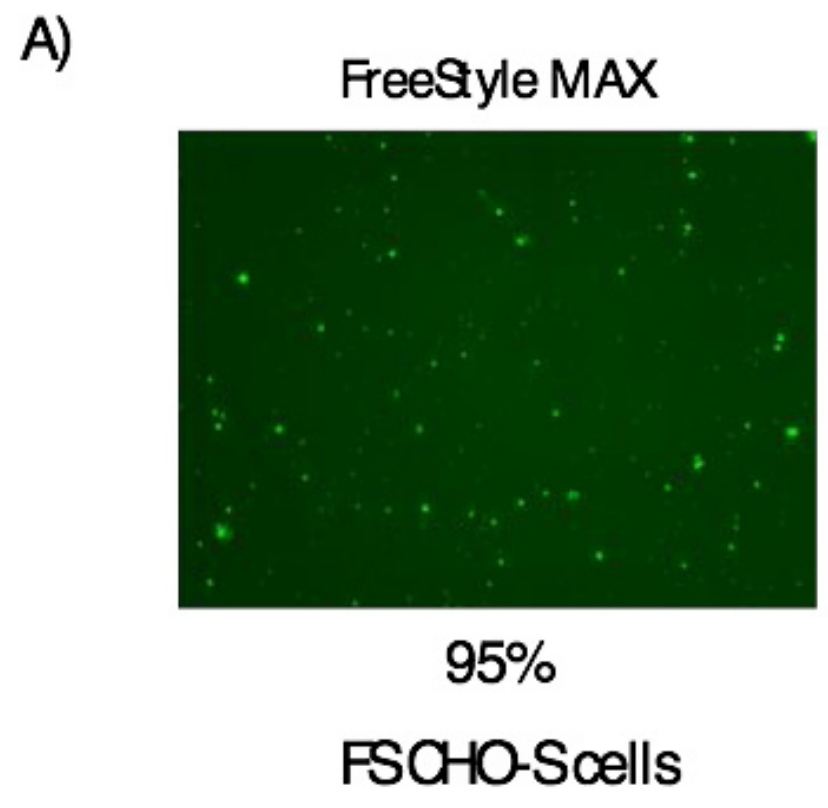

B)
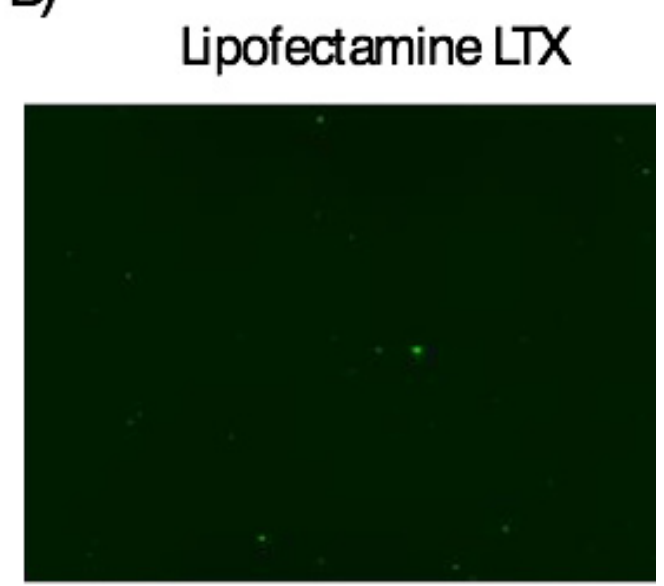

$21 \%$

NPLAd cells

Fig. (1). The transfection efficiency of FS CHO-S (A) and NPLAd cells (B).

\subsection{Preparation of Growth Control Medium}

NPLAd medium was found to be good for cell growth, but with low protein expression [13]. We modified the previously reported NPL medium [13] with the aim of maintaining the survival of cells at high density. The improved NPL medium was named NPLTT medium (NPL Medium for Transient Transfection). In NPLTT medium, the concentrations of amino acids were increased as a protein expression resource. The concentrations of sugars were increased as an energy source for cells. Antioxidant reagents, which were ascorbic acid and glutathione were added to suppress cell damage. In addition, 1\% Pluronic F-68 Non-ionic Surfactant (Thermo Fisher Scientific Inc.) was added to suppress shock absorption of cells during shaking culture and form cell clumps. In NPLAd system, valproic acid (VPA) was added to medium after transfection to inhibit histone deacetylase activity [15], suppresses gene uptake and cell proliferation [16].

\subsection{Comparison of Cell Growth and Antibody Expression Levels in NPLAd and Free Style Systems.}

In transient expression system, cell growth was not necessary for protein expression because most of cells were not transfected by expression vector. Keeping this in view, we developed low growth rate medium for antibody expression. To confirm the characterization of our developed expression system, we compared of cell growth and antibody expression levels in NPLAd system to Free style system, which was one of the commercially available useful and high expression systems. The time course of cell numbers is shown in Fig. (2A). In the case of Free style system, cell numbers were increased time dependently until 7 days. After that, cell numbers were dramatically decreased. After 12 days, cell numbers were decreased to almost seeding numbers. This cell number decrease could be over cell growth. On the other hand, in case of NPLAd system, cell numbers were not increased until 10 days, after that they were slightly 
decreased. We seeded cells almost confluent, because NPLTT medium did not support cell growth.

The time course of antibody expression levels is shown in Fig. (2B). In the case of Free style system, antibody expression level was increased time dependently from 4 to 7 days, and reached maximum to approximately $2500 \mathrm{ng} / \mathrm{ml}$, after that the expression level was decreased. In NPLAd system, expression level of anti-HER2 antibody was increased until 14 days, and reached maximum to approximately $4500 \mathrm{ng} / \mathrm{ml}$. The results indicated that expression level in NPLAd system was 1.8 times higher than that in Free style system. After 7 days, the expression level in NPLAd system was 1.4 times higher than that in Free style system. In NPLAd system, high numbers of transfected cells could be kept without cell growth within 10 days. These high numbers of cells could express high level of anti-HER2 antibody.
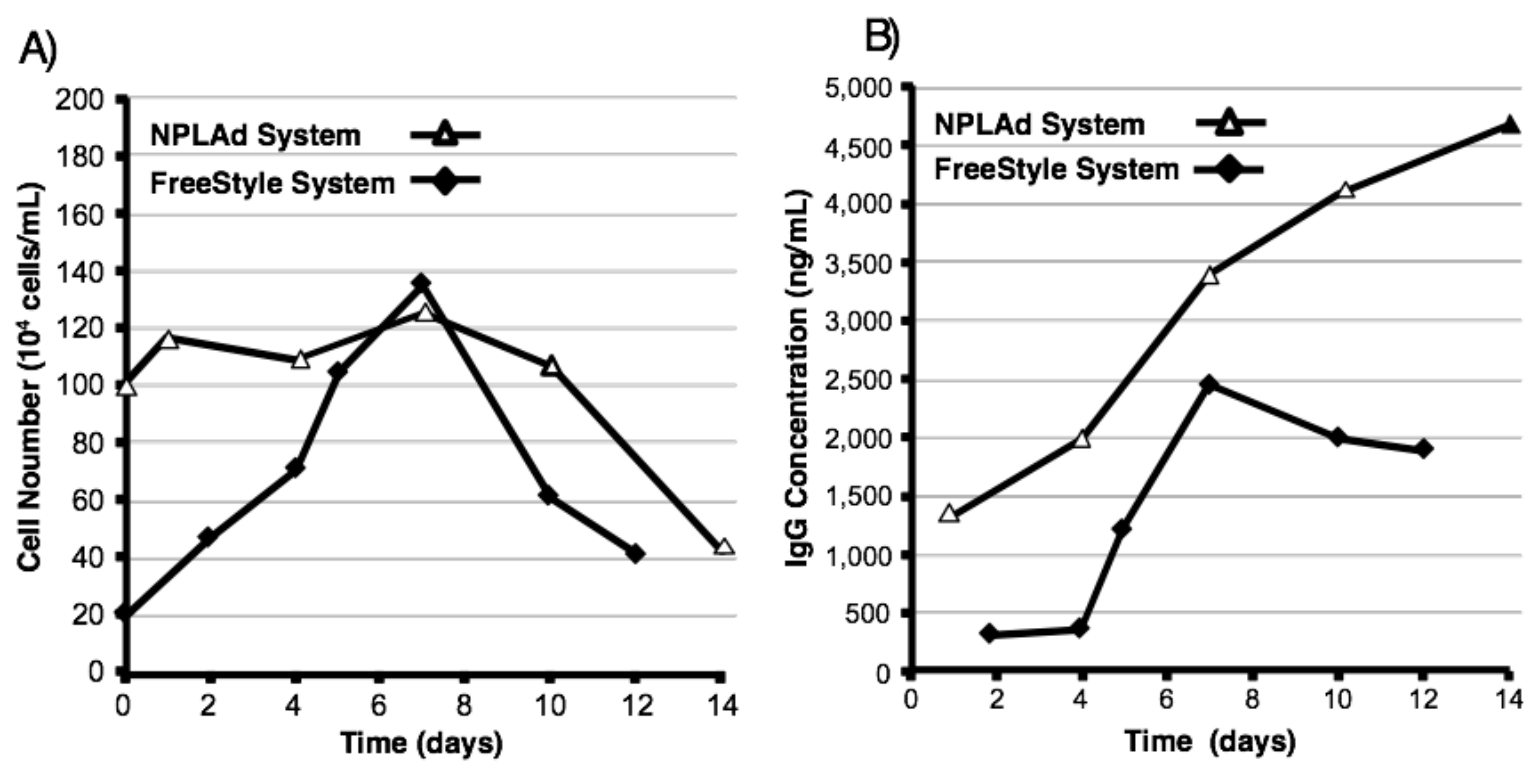

Fig. (2). Comparison of cell growth (A) and antibody expression levels (B) in NPLAd and Free style systems.

\subsection{Purification of Recombinant Antibodies}

Antibody recovery rate by Protein A affinity chromatography was 70\% or more (NPLAd System 73.3\%, FreeStyle System $84.8 \%$ ), antibody purity was $90 \%$ or more (NPLAd System 92\%, FreeStyle System 90\%). In NPLAd System, NPLTT medium contains almost no protein so antibody protein can be purified to high purity only by concentration and affinity chromatography.

As a result of Western blot after purification, three bands reacting with anti-human IgG were confirmed between approximately 100 and $150 \mathrm{KDa}$ in molecular weight. We estimated that the top band showed full-IgG (147 KDa), the middle band showed IgG without one right chain $(122 \mathrm{KDa})$ and the bottom band showed IgG without two right chains (97 KDa). The migration distance of each bands of antibodies expressed by NPLAd System and FreeStyle System were almost the same (Fig. 3).

\subsection{Comparison of Binding Activities of Recombinant Antibodies}

To confirm the antibody performance expressed by NPLAd system, we compared the binding activities of recombinant antibodies with Free style system. The A431 cells, HER2-expressed cell line [17], were seeded in a 96well plate. And then the anti-HER2 antibodies, which were expressed by NPLAd and Free style system, were added. The binding levels of recombinant antibodies were detected by human IgG ELISA. As shown in Fig. (4), binding activities of recombinant antibodies expressed by NPLAd and Free style system were almost the same. The results indicated that the antibody performance expressed by NPLAd system was almost the same to the one expressed by Free style system. 


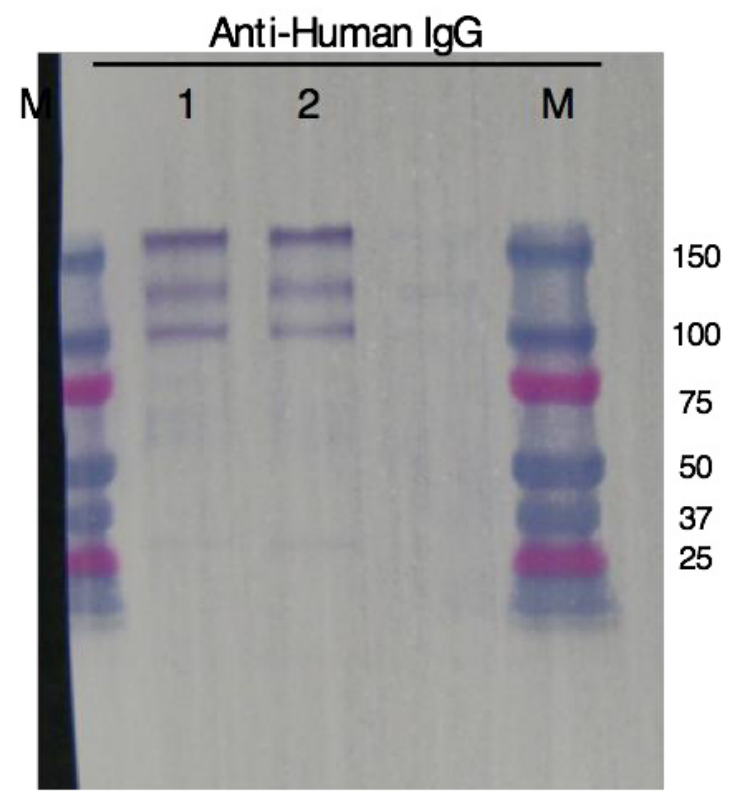

Fig. (3). Western blot analysis of recombinant antibody expressed by NPLAd and Free style systems. In SDS-PAGE, the sample was electrophoresed without reduction with DTT. After electrophoresis, Western blotting and immunostaining with anti-human IgG-HRP were performed. For electrophoresis, molecular weight is estimated using MW 10-150 kDa marker. Lane 1 is purified antibody of NPLAd System (97 ng), lane 2 is purified antibody of Free style system (81 ng). Lane M is the Precision Plus Protein Dual Color Standards Marker, which shows a molecular weight range of 10-150 kDa.

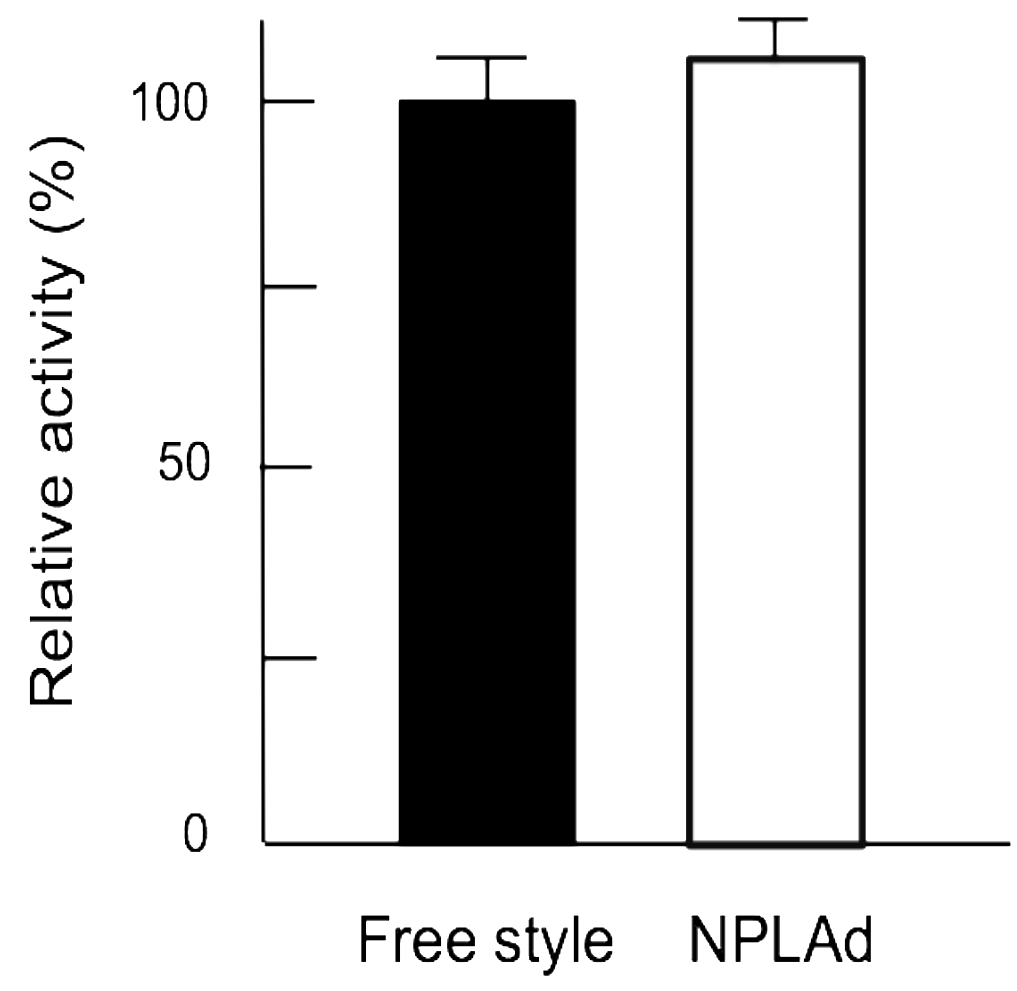

Fig. (4). Relative binding activities of antibodies expressed by NPLAd and Free style systems. $50 \mathrm{ng} / \mathrm{ml}$ of antibodies were used for assay. $\mathrm{N}=3$.

\section{CONCLUSION}

We developed high antibody expression system namely NPLAd system, which used low growth rate medium. Even at low transfection efficiency, antibody expression level of NPLAd system was 1.4 times higher than that in Free style system. We concluded that our developed growth controlled medium enhanced recombinant antibody expression level. 
This expression system would be useful for recombinant protein expression.

\section{ETHICS APPROVAL AND CONSENT TO PARTICIPATE}

Not applicable.

\section{HUMAN AND ANIMAL RIGHTS}

No Animals/Humans were used for studies of this research.

\section{CONSENT FOR PUBLICATION}

Not applicable.

\section{CONFLICT OF INTEREST}

The authors declare no conflict of interest, financial or otherwise.

\section{ACKNOWLEDGEMENTS}

Declared none.

\section{REFERENCES}

[1] Obradovic M, Mrhar A, Kos M. Market uptake of biologic and small-molecule--targeted oncology drugs in Europe. Clin Ther 2009; 31(12): 2940-52.

[http://dx.doi.org/10.1016/j.clinthera.2009.12.019] [PMID: 20110034]

[2] Maggon K. Monoclonal antibody "gold rush". Curr Med Chem 2007; 14(18): 1978-87. [http://dx.doi.org/10.2174/092986707781368504] [PMID: 17691940]

[3] Aggarwal RS. What's fueling the biotech engine-2012 to 2013. Nat Biotechnol 2014; 32(1): 32-9. [http://dx.doi.org/10.1038/nbt.2794] [PMID: 24406926]

[4] Ecker DM, Jones SD, Levine HL. The therapeutic monoclonal antibody market. MAbs 2015; 7(1): 9-14. [http://dx.doi.org/10.4161/19420862.2015.989042] [PMID: 25529996]

[5] Frenzel A, Hust M, Schirrmann T. Expression of recombinant antibodies. Front Immunol 2013; 4: 217. [http://dx.doi.org/10.3389/fimmu.2013.00217] [PMID: 23908655]

[6] Aghajani J, Mirtajani SB, Kojuri SA, Zaheire R, Ayoubi S. Assessment of HIV infection in cells of infected individuals. Banat's J Biotechnol 2017; 8: 24-38.

[http://dx.doi.org/10.7904/2068-4738-VIII(16)-24]

[7] Azar OL, Moradi Kor N, Ehsani M, Aiubi S, Rahmani FA. Cytochemical staining for the detection of acute and chronic blood leukemia. Banat's J Biotechnol 2016; 7: 46-52. [http://dx.doi.org/10.7904/2068-4738-VII(14)-46]

[8] Kelley B. Industrialization of mAb production technology: The bioprocessing industry at a crossroads. MAbs 2009; 1(5): 443-52. [http://dx.doi.org/10.4161/mabs.1.5.9448] [PMID: 20065641]

[9] Kunert R, Reinhart D. Advances in recombinant antibody manufacturing. Appl Microbiol Biotechnol 2016; 100(8): 3451-61 [http://dx.doi.org/10.1007/s00253-016-7388-9] [PMID: 26936774]

[10] Ahmadi S, Davami F, Davoudi N, et al. Monoclonal antibodies expression improvement in CHO cells by PiggyBac transposition regarding vectors ratios and design. PLoS One 2017; 12(6): e0179902. [http://dx.doi.org/10.1371/journal.pone.0179902] [PMID: 28662065]

[11] Sunstrom NA, Gay RD, Wong DC, Kitchen NA, DeBoer L, Gray PP. Insulin-like growth factor-I and transferrin mediate growth and survival of Chinese hamster ovary cells. Biotechnol Prog 2000; 16(5): 698-702. [http://dx.doi.org/10.1021/bp000102t] [PMID: 11027159]

[12] Fisher DA. Epidermal growth factor in the developing mammal. Mead Johnson Symp Perinat Dev Med 1988; 33-40. [PMID: 2485437]

[13] Sasaki T, Taniguchi A. Development of a non-protein and lipid medium adopted cell line for biopharmaceutical recombinant protein expression. Open Biotechnol J 2013; 7: 1-6. [http://dx.doi.org/10.2174/1874070701307010001]

[14] Sandbichler AM, Aschberger T, Pelster B. A method to evaluate the efficiency of transfection reagents in an adherent zebrafish cell line. Biores Open Access 2013; 2(1): 20-7. [http://dx.doi.org/10.1089/biores.2012.0287] [PMID: 23515475]

[15] Phiel CJ, Zhang F, Huang EY, Guenther MG, Lazar MA, Klein PS. Histone deacetylase is a direct target of valproic acid, a potent 
anticonvulsant, mood stabilizer, and teratogen. J Biol Chem 2001; 276(39): 36734-41.

[http://dx.doi.org/10.1074/jbc.M101287200] [PMID: 11473107]

[16] Wulhfard S, Baldi L, et al. Valproic acid enhances recombinant mRNA and protein levels in transiently transfected Chinese hamster ovary cells. J Biotechnol 2010; 20; 148(2-3): 128-32.

[17] McCluskey AJ, Olive AJ, Starnbach MN, Collier RJ. Targeting HER2-positive cancer cells with receptor-redirected anthrax protective antigen. Mol Oncol 2013; 7(3): 440-51.

[http://dx.doi.org/10.1016/j.molonc.2012.12.003] [PMID: 23290417]

\section{(C) 2018 Phuc et al.}

This is an open access article distributed under the terms of the Creative Commons Attribution 4.0 International Public License (CC-BY 4.0), a copy of which is available at: (https://creativecommons.org/licenses/by/4.0/legalcode). This license permits unrestricted use, distribution, and reproduction in any medium, provided the original author and source are credited. 